\title{
SimulNet: Virtual tele-laboratories over the Internet
}

\author{
M.Llamas, L. Anido and M.J. Fernández \\ Área de Ingeniería Telemática \\ Departamento de Tecnologías de las Comunicaciones \\ E.T.S.I. Telecomunicación. Universidade de Vigo \\ Campus Universitario 36200 Vigo. SPAIN \\ e-mail :\{martin,lanido,manolo\}@ait.uvigo.es
}

\begin{abstract}
Teaching and education have changed to meet the needs of the present-day working market. Because of this, professional and practical training are absolutely essential to educate good professionals, especially in the scope of Technology and Science. It is not easy to achieve this objective in distance learning environments. Our virtual tele-laboratory fills the gap between theoretical and practical teaching in a distance learning environment based on the Internet and the WWW.

SimulNet is a distributed, remote access computer based training (CBT) system. Unlike other teleteaching systems whose aim is to achieve a virtual classroom, SimulNet provides a virtual laboratory to put theoretical knowledge into practice. This is done by delivering software through the Internet which can be run on any computer. These distributed applications are simulators of those tools which can be found in a conventional laboratory. The system also provides an on-line communication channel between students and teachers to follow students' traces in their interaction with the simulator and to assist students. These features allow us to achieve a virtual laboratory where drawbacks due to geographical separation between teachers and students have overcome and advantages of telelearning and teleteaching have been gathered.
\end{abstract}

Keywords

Simulation, CBT, Teleteaching, Java, WWW, Training 


\section{INTRODUCTION}

The ASTRO Development Team from Vigo University, has implemented a computer based training (CBT) system based on $\mathrm{WWW}^{*}$ that permits supervised execution of courses, trainee evaluation and also maintains trainee state, which depends on his/her previous actions, selected navigation mode and obtained scores. ASTRO (ASTRO, Llamas 1996) allows students or trainees to follow different courses which are composed of a structured set of hypermedia documents. In this way, trainees are able to follow theoretical courses through the Internet.

The next step is to provide an approach to a virtual laboratory where the acquired knowledge can be put into practice as University or Secondary School students do in their conventional training laboratories.

SimulNet sends through the Internet applications which can be run on students' computers. So, avoiding the network overhead, students run conventional laboratory simulators interactively. The key to achieving this high level of interaction, essential in a tele-laboratory, is that SimulNet is implemented solely using Java.

Java (Abdel 1996, Java, Kramer 1996), developed by Sun Microsystems, is an object-oriented language for the Internet. Among its features, one of the most important is platform independence. Therefore, SimulNet, client and server applications, may be run on any computer, whatever its operating system or architecture.

\section{WHERE AND WHY COULD WE USE SIMULNET?}

Teaching and education have changed to meet the needs of present-day working market. Because of this, professional and practical training are essential to train good professionals, especially in the scope of Technology and Science. It is not easy to achieve this objective in a distance learning environment based on the Internet and the WWW.

A university, as the last step in student education, is the natural place to use a system like this. SimulNet allows students do their laboratories at home, wherever they live, and at the same time, it solves the problems related to overcrowding: restrictions due to space, allocation of resources, etc. A suitable timetable could let students go to their faculty or school only few days a week. We expect that students at Vigo University will be able to use SimulNet in several laboratories related to computer architecture subjects the following school year. Apart from a university, a virtual tele-laboratory system may be used in polytechnics and secondary schools.

\footnotetext{
* World Wide Web, http://www.w3.org
} 
On the other hand, those academic institutions which have been traditionally related to distance learning, can broaden their offerings using the SimulNet system. The Internet, is an ideal way to transmit knowledge. Our system would allow students to put the acquired knowledge into practice directly and learn by teletraining.

\section{OBJECTIVE: VIRTUAL TELE-LABORATORY}

The Internet and the World Wide Web are distributed environments inherently. A virtual tele-laboratory over them needs a set of additional facilities to overcome the physical separation and give students and teachers the same feeling as if they were in the laboratory itself.

SimulNet provides several functions to achieve this objective:

- The simulators are interactive. There is no network overhead as the simulators run on the student's own computer.

- Trainee traces reflecting their interaction with the simulator are sent to their tutor straight away, and are stored in the SimulNet host server to be looked over afterwards.

- An on-line communication channel between teachers and students is provided. The main purpose is to :utor students in the use and aim of the laboratory's simulator. This channel can be used by the different communication facilities that SimulNet implements:

- Text talk between a student and his/her tutor. This can be used to explain student's doubts or help students in their interaction with the simulator.

- Multi-talk among several students and their tutor. This can be very useful to solve students' questions or teach any topic related to the current training practice.

- Virtual blackboard shared among several students and their tutor. The blackboard's contents are displayed identically on every other blackboard. There are two operation modes: free mode, where the tutor and the students can present graphical information without any restrictions; and master-slave mode, where the tutor uses the blackboard for his/her explanations without interference from students.

- Electronic mail between students and tutors of SimulNet. This can be used by tutors to send training practice instructions and by students to return their results and conclusions.

- Notebooks (Lai 1995). Its purpose is to allow teachers to share notes about students and can be used to store students' grades.

- SimulNet overcomes the physical boundaries of a conventional laboratory and the time constraints of a course timetable. However, it provides the possibility of establishing a timetable which could be needed due to organisational reasons 
or to occasionally restrict students' free access. SimulNet also provides facilities for substitutions among teachers or extra tutorings.

- It registers the entries and exits into and out of the system.

- Students and tutors can choose among several languages to interact with the system in every teleteaching session.

\section{USER INTERACTION}

For the sake of simplicity we are using from this point the masculine to refer to trainees or tutors. Of course, the reader must assume they could be men or women without distinction.

When a trainee/tutor begins a SimulNet telelearning/teleteaching session, first of all he can choose among several languages to be used in the user interface and then he must be successfully identified. After that, a list of those simulators he is allowed to access to is presented. At his point the tutor/trainee selects a simulator and the educational session starts. If he has any message stored in his mailbox it would be sent to him.

\subsection{Trainee interaction}

Trainees run simulators on their own computers achieving a high level of interaction. Any outstanding action from a teaching point of view would be sent to the trainee's tutor.

Trainees have on-line and off-line learning tools at their service. They are allowed to use off-line tools, like the internal e-mail, at any moment to send any question or comment to his tutor or to the system manager. On-line teaching tools, like text-talk or the virtual blackboard can be used by trainees in a more restrictive way, they are only allowed to send requests to their tutors who have the final decision to establish, for instance, a multi-talk or virtual blackboard session. From a conventional teaching point of view, students should ask to be allowed to speak before doing it.

\subsection{Tutor interaction}

Tutors can be connected to SimulNet without following trainees' traces or receiving trainees' questions and requests. In this way, they can look over previous trainees' traces, use the notebook facility or use the internal e-mail to send training practice instructions without interferences from trainees. At any moment they can decide to start their tutoring session and therefore receive trainees' traces and requests. 
Unlike trainees, tutors can use on-line and off-line teaching tools at their service with no restriction. They can involve any trainee in any teaching tool to solve any doubt or teach any topic related to the training practices.

Moreover, they are given access to their trainees' traces as they do the training practice. So, tutors can find out the most common difficulties and help any trainee who is presumed to be in trouble.

\subsection{An example}

In this section we shall show an example of a SimulNet teleteaching session which gives a good indication about the environment that will be achieved.

In a computer architecture course, for instance, the use of a real computer is essential to put theoretical knowledge into practice. We can use SimulNet to obtain the same objective in a teleteaching environment.

As usual in conventional teaching, the course may be scheduled over several practical sessions. Let's follow one of them. First of all, the teacher would enter into SimulNet and, before starting the teleteaching session, he could write the training practice instructions, for instance about addressing modes, and send them to students by means of the internal e-mail. Afterwards, he would start the tutoring session and see, by means of trainees' traces functionality, how his students entered the computer simulator and read the previously sent instructions. On the other hand, students, after reading the training practice instructions, would interact with the computer simulator or use any parallel CBT course system to go through the needed theoretical knowledge to do this practice.

As students do the training practice, their tutor would receive their traces. Meanwhile, he could look over trainees' traces in previous training practices to evaluate their progress. If the tutor noticed that any of them has any problem he could use the text-talk facility to communicate with him and solve any questions related to the current training practice or to the use of the computer simulator. At the same time on students' side, any trainee in difficulty would send a request to his tutor to have a text-talk session, for instance, in order to solve his problem. The tutor would receive this request and decide, whether to start a text-talk session. During this communication, the tutor could realise that this problem could be common among his students and presume that many of them would have the same problem in the current training practice. Therefore, he would start a multi-talk session and involve every trainee in it. If necessary, they could also use the virtual blackboard to introduce some graphical information like flow charts to explain a particular addressing mode, for example.

Eventually, the trainees would complete the training practice. Then, they would send their conclusions about the training practice to the tutor by internal e-mail: what the advantages of every addressing mode are, when and where they should use every of them, etc. The tutor would grade trainees' training practices and store the grades and observations using the notebook facility.

This is just an example of the use of SimulNet in distance learning. There are many CBT systems whose aim is to teleteach which would need a way to let 
students undertake practice and exercises with the knowledge they have acquired. That is our main contribution to distance teaching/learning.

\section{SIMULNET MANAGEMENT AND MAINTENANCE}

The SimulNet management application is able to install automatically the system's server side by itself. This application provides every essential feature to manage the system: join new simulators, students or teachers to the system, shape the simulators' behaviour, disk quotas for students and tutors, test the integrity and congruence of the SimulNet's database and configuration files avoiding an undesirable system behaviour, provide user authentication, etc.

Simulators are organised in groups. So, a set up in groups of laboratories with features and interests in common is available to the system manager. This can be very useful to establish an organisation related to different University departments, research teams, or any other organisational needs because there is no interference among students and teachers from different groups.

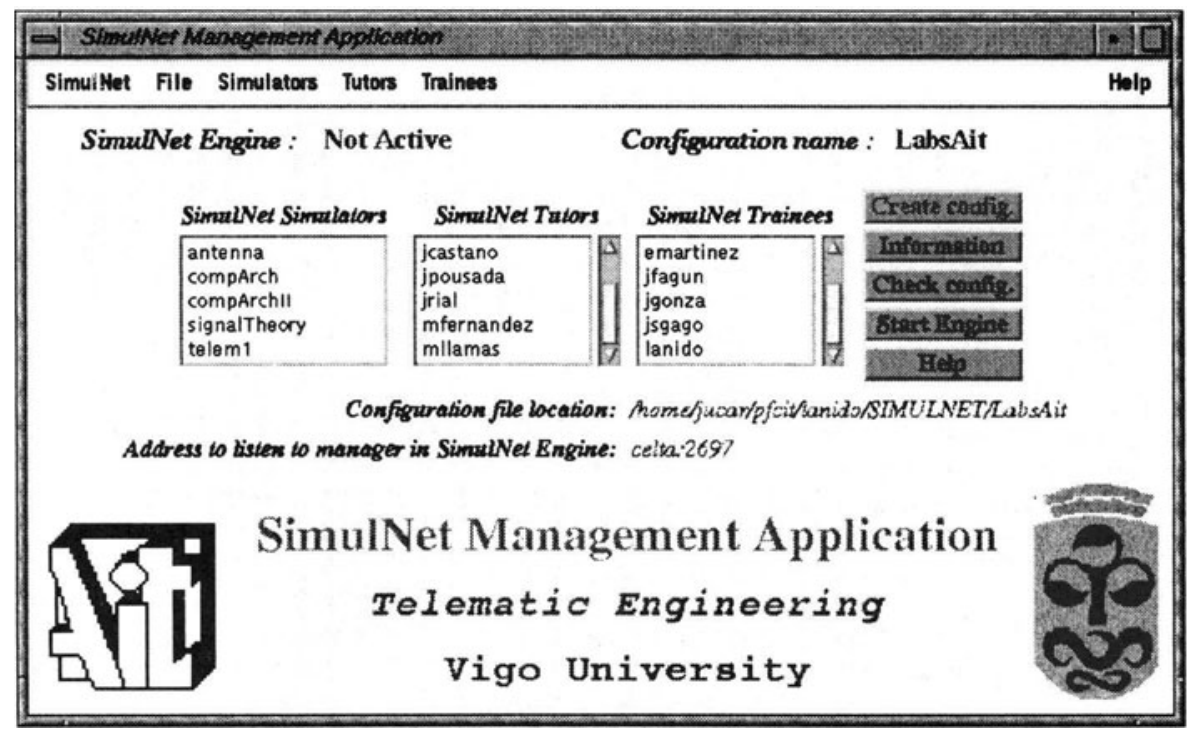

Figure 1. The SimulNet Management Application

The SimulNet management application provides an easy to use graphical user interface for the system manager to carry out his job, as shown in Figure 1. Without any knowledge about the underlying configuration files or data structures, he is able to manage the different groups of laboratories he is responsible for from the same management application. 


\section{ARCHITECTURE OVERVIEW}

SimulNet makes use of the Internet technology to implement the needed services. The system is composed of several applications which cooperate to implement each SimulNet facility, all of them having been developed using Java, therefore platform independence, high level of interaction and easy use over the Internet is achieved. Communication among them is performed using Internet transport layer protocols (TCP/IP). This is the basis for our distributed educational environment, several Java application cooperating by means of TCP/IP communication. Moreover we have also taken advantage of other existing WWW elements: HTTP service, HTML, WWW browser and server. The interaction among the different elements of the system is outlined in Figure 2 and will be explained in the next sections.

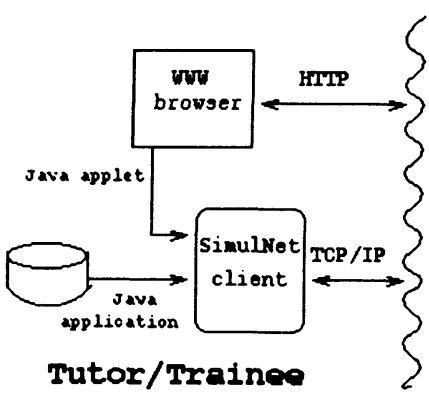

side

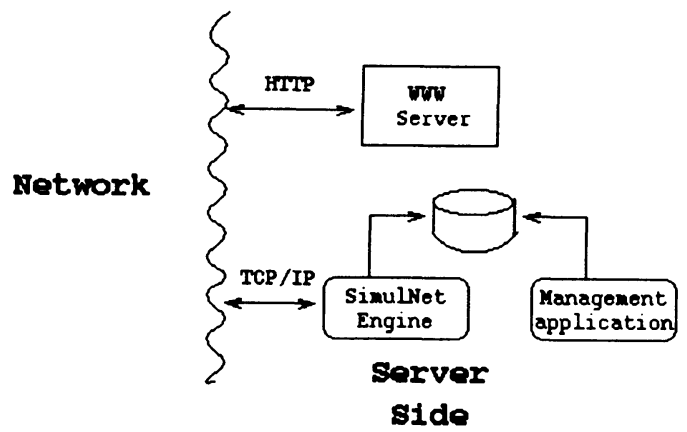

Figure 2. Module interaction.

\subsection{Tutor/trainee side}

Tutors and trainees can connect to SimulNet in two different ways:

1. By means of a standard WWW browser which would be used as a client browser to present an HTML document provided by the WWW server at the server side. This document contains an embedded Java applet which would launch and terminate any other Java application provided by the server side through the Internet.

2. Using a fully independent Java application stored at the tutor/trainee side. In this case, the SimulNet simulators could be used in a stand-alone way, i.e. trainees are able to practice without any help or supervision by his/her tutor. At the same time, the tutor/trainee can connect the Java application to the server side to benefit from the virtual laboratory advantages: communication channel, trainees' traces, etc. 
The two previous connection modes have different advantages. The first one, which is based on Java applets, needs no additional software, apart from the browser itself, at the tutor/trainee side. Therefore, any common Internet connection supplied by usual Internet-provider companies is enough. Nevertheless, due to security restrictions in the Java's implementation, trainees/tutors could only store data in the server host machine, not at tutor/trainee side. The second one overcomes this inconvenience as it is based on Java applications. So, storage capability at tutor/trainee side is provided. The only requirement is that the trainees/tutors' computers support the Java software.

Anyway, both provide all SimulNet functionalities as explained in the previous sections, and the choice between them depends on the simulator's particular aim. They are outlined in Figure 2.

\subsection{Server side}

The server side functionality is implemented by three different applications.

1. WWW server. Its function is to provide HTML documents which contain the SimulNet applets and support, in this way, the first trainee/tutor connection mode, as explained before. Communication to the WWW server is based on HTTP protocol as shown in Figure 2.

2. The SimulNet Management Application. This has been implemented solely using Java and carries out every trainees/tutors management functionality and simulators' configuration, see Figure 1. Every SimulNet feature is set up using this application and is communicated to the SimulNet Engine, whose function is explained below, by means of a twofold way: using TCP/IP and sharing the same file system, as is outlined in Figure 3.

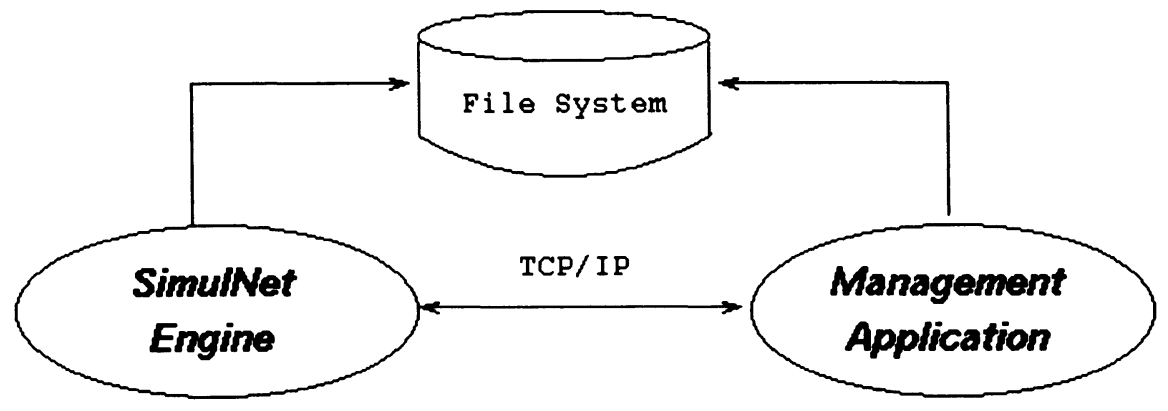

Figure 3. Communication between Engine and Management application.

3. The SimulNet Engine. This Java application supports the functionality of our system. It carries out the main system's behaviour which is set up using the management application. As shown in Figure 3, the Engine takes orders from it and performs any needed action to fit the system's behaviour to the new situation. So, new tutors or trainees could be imposed, etc, without stopping the 
system. Moreover it sends information about the tutors and trainees connected to the system to the management application.

To access a SimulNet simulator, tutors/trainees have to be properly identified by the system. The aim of this identification process is twofold: reject unknown (unregistered) SimulNet users and prevent tutors/trainees accessing simulators they are not allowed to. This user authentication is also provided by the SimulNet Engine.

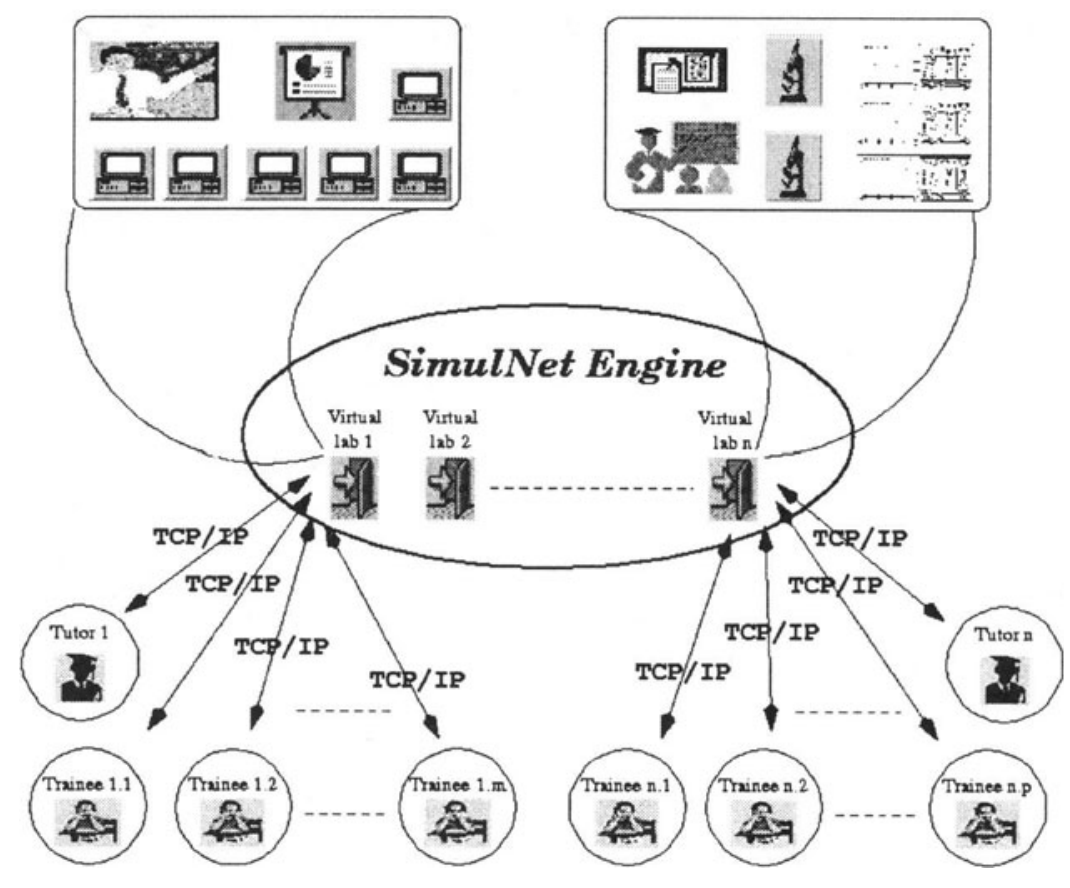

Figure 4. SimulNet architecture overview.

The SimulNet Engine provides a virtual communication channel between tutors and trainees by means of a twofold physical channel: tutor/trainee - SimulNet Engine and SimulNet Engine - trainee/tutor. Therefore, as we show in Figure 4, the TCP/IP communication between SimulNet Engine and trainees/tutors provides a virtual communication channel between them with the aim of achieving a real laboratory atmosphere as shown at the top of the figure.

This part of the server side is also responsible for managing the users' data storage and trainees' traces. At the same time that tutors and trainees are allowed to store files in the computer where the SimulNet Engine runs. The Engine itself ensures they do not exceed their disk quotas. On the other hand, trainees' traces 
are stored by the SimulNet Engine which sends them straight away to the tutor responsible for the trainee. So, every trainee's action can be supervised by his tutor and inspected afterwards.

Several simulators, which constitute a group of virtual laboratories, with their own trainees and tutors, can be supported by a unique SimulNet Engine. There is no interference among tutors or trainees from different simulators in their interaction with the system. This aspect is also shown in Figure 4, where several laboratories are outlined.

\section{CONCLUSIONS}

Java's features broaden the facilities that teleteaching/telelearning systems can provide. SimulNet has taken advantage of them and supplies a new point of view to distance learning and teaching: it allows students to do training practices in a virtual tele-laboratory. In this way, students and teachers are prevented from wasting time and money on travel.

The main objective of our system is to achieve a virtual laboratory, but despite this, it is open and flexible enough to provide different applications apart from those related to telelearning and teleteaching. This system can be used as a software on demand platform, where different applications (the simulators) are sent to the users hrough the Internet and where, if necessary, their execution could be supervised. Of course, every SimulNet facility could be used: use authentication, user's traces, communication channel among users, etc.

At the moment, this system is under development and we are implementing the ideas presented previously and, at the same time, we are looking for new facilities to improve the virtual laboratory atmosphere. We think this document offers a good idea of what will be achieved with the proposed framework.

This work is being partially supported by the University of Vigo in the framework of the "Apoio á innovación educativa" (support to innovation in education) program, project "ETLAO: Entorno de TeleLaboratorio para Arquitectura de Ordenadores" (Telelaboratory environment for Computer Architecture).

\section{REFERENCES}

Abdel, H., Kvande, B. and Nanjangud, S. (1996) Using Java for Multimedia Collaborative Applications. 3rd International Workshop on Protocols for Multimedia Systems (PROMS'96), Madrid.

ASTRO Course Engine Development Team. ASTRO Course Engine Public WWW

Server. http://astro.ait.uvigo.es/astrodocs/main.html

Java site. http://www.javasoft.com

Kramer, D. (1996) The Java Platform. A white paper. 
Lai, M., Chen, B. and Yuan S. (1995) Toward a New Educational Environment. Fourth International World Wide Web Conference. Boston

Llamas, M., Fernández, M.J., Gil, A., Rodríguez, R. and Suárez, A. (1996) A Costeffective Approach to WWW Education and Training. 3rd International Workshop on Protocols for Multimedia Systems (PROMS'96), Madrid.

\section{BIOGRAPHIES}

Martín Llamas received the Telecommunication Engineering (1986) and Doctor in Telecommunications (1994) degrees from the Universidad Politécnica de Madrid, Spain.

Luis E. Anido has recently graduated (1997) from Universidade de Vigo, Spain with a Telecommunication Engineering degree.

Manuel J. Fernández graduated from the Universidade de Santiago de Compostela, Spain with a Telecommunication Engineering degree in 1990, and from Universidade de Vigo with a Doctor in Telecommunications degree in 1997.

They joined the Telecommunication Engineering faculty of the Universidade de Vigo. In addition to teaching, they are involved in research in the areas of Formal Description Techniques and Computer Based Training. 